\title{
Role of pertuzumab in the treatment of HER2-positive breast cancer
}

\author{
This article was published in the following Dove Press journal: \\ Breast Cancer:Targets and Therapy \\ 26 May 2012 \\ Number of times this article has been viewed
}

\author{
Michael Hubalek \\ Christine Brantner \\ Christian Marth \\ Brustgesundheitzentrum Tirol, \\ Department of Obstetrics and \\ Gynecology, Innsbruck Medical \\ University, Innsbruck, Austria
}

Correspondence: Michael Hubalek Brustgesundheitzentrum Tirol, Department of Obstetrics and Gynecology, Medical University Innsbruck, Anichstrasse 35, A-6020 Innsbruck, Austria

$\mathrm{Tel}+4351250481285$

Fax +43 51250423050

Email michael.hubalek@i-med.ac.at

\begin{abstract}
Pertuzumab, a humanized monoclonal antibody to the HER2 receptor, represents a promising new anti-HER2 agent with a novel mechanism of action targeting the inhibition of HER2 dimerization. Nonclinical and clinical data to date indicate that pertuzumab provides a broader HER2 blockade through the inhibition of HER2 heterodimerization. In preclinical experiments, pertuzumab has demonstrated superior antitumor effects when combined with other anti-HER2 treatments such as trastuzumab, compared to when used as monotherapy. Trastuzumab and pertuzumab monoclonal antibodies bind to distinct epitopes on the HER2 receptor without competing with each other, resulting in distinctive mechanisms for disrupting HER2 signaling. These mechanisms are complementary and result in augmented therapeutic efficacy when pertuzumab and trastuzumab are given in combination. Clinically, pertuzumab may have optimal therapeutic effects when given to patients with HER2-positive cancers, in combination with trastuzumab. This observation is supported by recent clinical trials in the metastatic as well as neoadjuvant setting. Intravenous pertuzumab had an acceptable tolerability profile when added to trastuzumab and chemotherapy. This overview will review recent advances in the clinical development of this HER2-targeted therapy.
\end{abstract}

Keywords: HER2, breast cancer, pertuzumab, trastuzumab

\section{Introduction}

Overexpression of HER2 in breast cancer is correlated with high histologic grade, increased mitotic activity, $p 53$ mutation, negative estrogen receptor (ER) status, absence of $b c l 2$, and absence of lobular architecture. Despite associations with other known negative prognostic factors, human epidermal growth factor receptor-2 (HER2) overexpression is independently associated with poorer disease-free survival and overall survival (OS) compared with tumors that do not overexpress HER2. ${ }^{1}$

Targeting the human epidermal growth factor receptor (HER) family of receptor tyrosine kinases has proven to be effective in cancer therapy. Trastuzumab, an monoclonal antibody that binds to the extracellular domain of the HER2 receptor, has become a standard treatment option for women with HER2 overexpression. ${ }^{2}$ Various studies on adjuvant as well as metastatic breast cancer have proved the efficacy of this antibody.,4 Yet, despite the advances that have come with the introduction of this novel therapy, there are still a number of HER2-positive breast cancers that exhibit intrinsic (primary) or de novo (secondary) resistance to HER2-targeted therapy. ${ }^{5}$ As a consequence, new anti-HER2 agents are currently under development. One of the most promising is the monoclonal antibody, pertuzumab. 


\section{Pertuzumab: mechanisms of action}

Pertuzumab (rhuMAb 2C4) is a fully humanized monoclonal antibody that is based on the human immunoglobulin (Ig) G1( $\kappa)$ framework sequences. This antibody acts by blocking the association of HER2 with other HER family members, including epidermal growth factor receptor (EGFR, also known as HER1), HER3, and HER4. As a result, the formation of HER heterodimers is inhibited. ${ }^{6,7}$ Pertuzumab represents a new class of drugs that targets HER2 dimerization. Like trastuzumab, pertuzumab is a recombinant humanized monoclonal antibody that binds to the extracellular domain of HER2. It consists of two 449 residue heavy chains and two 214 residue light chains. Pertuzumab binds to an epitope in domain II, the dimerization domain of the HER 2 extracellular domain, which is distinct from the binding site of trastuzumab in domain IV (Figure 1) ${ }^{8,9}$ Due to their complimentary modes of action, there is strong rationale for the combination of pertuzumab with trastuzumab for the treatment of HER2 overexpressing disease.

In vitro, pertuzumab blocks heregulin (HRG)-induced activation of the phosphatidylinositol-3-kinase (PI3 K) cell survival pathway, whereas trastuzumab does not. This suggests that pertuzumab is superior to trastuzumab in blocking ligand-activated HER2 signaling. In the breast

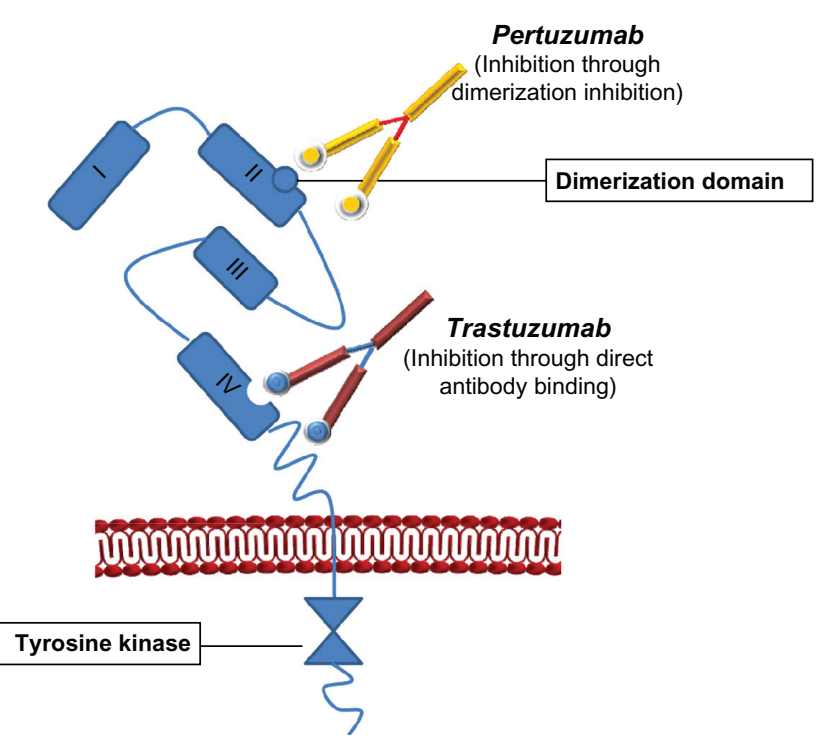

Figure I Pertuzumab is a fully humanized monoclonal antibody based on the human $\operatorname{lgGI}(\kappa)$ framework sequences that consists of two heavy chains (449 residues) and two light chains ( 214 residues).

Notes: Like trastuzumab, pertuzumab is directed against the extracellular domain of HER2. However, it differs from trastuzumab in the epitope-binding regions of the light chain ( 12 amino acid differences) and heavy chain ( 29 amino acid differences). As a result, pertuzumab binds to an epitope within what is known as subdomain 2 of HER2 while the epitope for trastuzumab is localized to subdomain 4. Pertuzumab inhibits tumor growth by sterically blocking the formation of HER2 ligand-receptor complexes. The formation of heterodimers is inhibited (eg, HER2-HER3, HER2-HER4 heterodimers, etc). The antibody blocks ligand-activated HER2 signaling, whereas trastuzumab does not. carcinoma cell line MCF-7, pertuzumab blocks HRG-induced activation of the PI3 K cell survival pathway - as indicated by a lack of phosphorylation of a key enzyme (Akt) in this pathway - while trastuzumab does not. ${ }^{10}$ Additionally, pertuzumab activates antibody-dependent cellular cytotoxicity (ADCC) with the same potency as trastuzumab. ${ }^{11}$ In human tumor xenograft models in mice, single agent pertuzumab was active against various tumors (including those without HER2 overexpression) such as lung, breast, ovarian, and prostate cancers. ${ }^{10}$ Pertuzumab augmented the antitumor effect of various cytotoxic drugs representing different mechanisms of action, as well as other HER pathway inhibitors such as the HER1-targeting agent, erlotinib. ${ }^{12}$ In HER2-positive xenograft models, pertuzumab demonstrated synergistic antitumor activity in combination with trastuzumab or trastuzumab DM-1 (T-DM1). ${ }^{11}, 13$ The following sections will discuss the development and clinical activity of pertuzumab.

\section{Clinical development}

Due to pertuzumab's distinct mode of action compared to trastuzumab, early clinical developments built confidence in the possibility of clinical activity in various types of cancers, even those that showed no HER2 amplification. In 2005, a Phase I trial of pertuzumab monotherapy was conducted in patients with advanced cancer. ${ }^{14}$ Most of these tumors were not overexpressing the HER2 receptor. In this evaluation, pertuzumab was well tolerated at all dose levels, and a maximum tolerated dose was not reached. Toxicity was generally mild as twelve grade $3 / 4$ events were reported, of which only six were thought to be related to the medication under examination. Symptoms included abdominal pain $(14 \%)$, nausea (5\%), vomiting (5\%), and diarrhea (5\%). Pharmacokinetic analyses of serum concentrations revealed that levels of trastuzumab rapidly declined over the first 2 to 3 days. The half-life of pertuzumab is 3 weeks and the volume of distribution approximated the serum volume. Pharmacokinetics obtained in the Phase I trial appeared to be similar to the preclinical data (see Table 1).${ }^{15}$ Early trials used a dose ranging from 0.5 to $15 \mathrm{mg} / \mathrm{kg}$ and systemic clearance remained constant. Based on these data, a dosing interval of 3 weeks was recommended for clinical application. In the Phase II studies, a loading dose of $840 \mathrm{mg}$ (followed by $420 \mathrm{mg}$ $\mathrm{q} 3 \mathrm{w}$ ), was capable of attaining steady-state trough and peak concentrations by the second cycle (see Table 1). ${ }^{16}$ Clinical activity was observed in patients with HER2 low-expressing tumors who had received pertuzumab either as a single agent or in combination with cytotoxic chemotherapy. Complete responses have not been observed in any of these trials. In 
Table I Features and properties of pertuzumab

\begin{tabular}{l} 
Clinically tested indications of pertuzumab for \\
the treatment of breast cancer (Phase II/III trials) \\
- First-line treatment of HER2-positive metastatic \\
breast cancer in combination with trastuzumab \\
and docetaxel \\
- Neoadjuvant treatment of locally advanced \\
breast cancer in combinations with trastuzumab \\
anthracyclines, docetaxel, carboplatin \\
Mechanisms of action \\
- Prevents dimerization of HER2 with other \\
ligand-activated HER receptors, inhibiting the \\
activation of intracellular signaling pathways \\
associated with the proliferation and survival \\
of cancer cells \\
- Stimulates antibody-dependent cell-mediated \\
cytotoxicity (ADCC) \\
Dosing in most clinical trials for breast cancer \\
Loading dose 840 mg \\
Maintenance dosage 420 mg every 3 weeks \\
Route of administration intravenous infusion \\
Pharmacokinetic profile of intravenous \\
pertuzumab 420 mg in patients with advanced \\
solid tumors ${ }^{14}$ \\
Mean maximum serum concentration \\
Mean area under the serum concentration-time \\
curve from time zero to infinity \\
Mean volume of distribution at steady state \\
Mean clearance \\
Mean elimination half-life \\
Adverse events (all grades) occurring in $\geq 5 \%$ more \\
pertuzumab than placebo recipients ${ }^{21}$ \\
Diarrhea, rash, mucosal inflammation, febrile \\
neutropenia, and dry skin \\
\hline
\end{tabular}

single agent pertuzumab studies, partial responses or stable disease lasting $\geq 6$ months have been observed in $15 \%$ of patients with ovarian cancer and in $8 \%$ of patients with HER2 low-expressing breast cancer. ${ }^{14,17}$ However, due to the limited efficacy observed in these studies, generally stable diseases of relatively short duration suggested that there would be little benefit in further investigating the use of single-agent pertuzumab in unselected patients with HER2-negative diseases. Further development of this compound is focused on women with HER2-overexpressing breast cancer.

\section{Pertuzumab in clinical trials for breast cancer}

In a study by Baselga et al, patients with advanced HER2positive breast cancer in whom disease progression had occurred during prior trastuzumab-based therapy, received trastuzumab weekly ( $4 \mathrm{mg} / \mathrm{kg}$ loading dose, then $2 \mathrm{mg} / \mathrm{kg}$ every week) or every 3 weeks ( $8 \mathrm{mg} / \mathrm{kg}$ loading dose, then $6 \mathrm{mg} / \mathrm{kg}$ every 3 weeks) and pertuzumab every 3 weeks $(840 \mathrm{mg}$ loading dose, then $420 \mathrm{mg}$ every 3 weeks). ${ }^{18}$ The Phase II trial (BO17929 trial) assessed the efficacy and safety profile of the combination in patients $(\mathrm{n}=66)$ with HER2-positive breast cancer whose disease had progressed during prior trastuzumab-based therapy (see Table 2). The objective response rate was $24.2 \%$, and the clinical benefit rate was $50 \%$. Five patients (7.6\%) experienced a complete response, eleven patients (16.7\%) experienced a partial response, and 17 patients $(25.8 \%)$ experienced stable disease for 6 or more months. Median progression-free survival (PFS) was 5.5 months. Overall, the combination of pertuzumab and trastuzumab was well tolerated, and AEs were mild to moderate.

A neoadjuvant Phase II study was initiated, based on this study. ${ }^{19}$ In the NEOSPHERE study, treatment-naive women with HER2-positive breast cancer were randomly assigned to four treatment groups $(1: 1: 1: 1)$. Depending on treatment group, patients were to receive four neoadjuvant cycles of: trastuzumab $(8 \mathrm{mg} / \mathrm{kg}$ loading dose, followed by $6 \mathrm{mg} / \mathrm{kg}$ every 3 weeks) plus docetaxel $\left(75 \mathrm{mg} / \mathrm{m}^{2}\right)$, escalating, if tolerated, to $100 \mathrm{mg} / \mathrm{m}^{2}$ every 3 weeks (group A), or pertuzumab (loading dose $840 \mathrm{mg}$, followed by $420 \mathrm{mg}$ every 3 weeks) and trastuzumab plus docetaxel (group B), or pertuzumab and trastuzumab (group C), or pertuzumab plus docetaxel (group D). The primary endpoint, examined in the intentionto-treat population, was pathological complete response $(\mathrm{pCR})$ in the breast. Of the 417 eligible patients, 107 were randomly assigned to group A, 107 to group B, 107 to group C, and 96 to group D. Patients who received pertuzumab and trastuzumab plus docetaxel (group B) had a significantly improved pathological complete response rate (49 of 107 patients; $45.8 \%$ [95\% CI: 36.1-55.7]) compared with those given trastuzumab plus docetaxel (group A; 31 of 107; 29.0\% [95\% CI: 20.6-38.5]; $P=0.0141)$. Twenty-three of $96(24.0 \%$ [95\% CI: 15.8-33.7]) women who received pertuzumab plus docetaxel (group D) had a pathological complete response, as did 18 of 107 (16.8\% [95\% CI: 10.3-25.3]) who received pertuzumab and trastuzumab (group C). Pathologic complete response was up to $63.2 \%$ with the combination of the two antibodies and docetaxel in the hormone receptor-negative subgroup.

The most common AEs of grade 3 or higher were neutropenia (61 of 107 women in group A, 48 of 107 in group B, one of 108 in group C, and 52 of 94 in group D), febrile neutropenia (eight, nine, none, and seven, respectively), and leucopenia (13, five, none, and seven, respectively). The number of serious AEs was similar in groups $\mathrm{A}, \mathrm{B}$, and $\mathrm{D}$ (15-20 serious AEs per group in $10 \%-17 \%$ of patients), 
Table 2 Completed clinical trials examining the efficacy of pertuzumab in breast cancer

\begin{tabular}{|c|c|c|c|c|c|}
\hline $\begin{array}{l}\text { Number of } \\
\text { patients }\end{array}$ & Population & Design & Treatment & Comments & Ref \\
\hline $\begin{array}{l}\mathrm{NCl}-06-\mathrm{C}-0035 \\
\mathrm{n}=12\end{array}$ & $\begin{array}{l}\text { HER2-positive } \\
\text { metastatic breast cancer } \\
\text { after standard therapy }\end{array}$ & $\begin{array}{l}\text { Phase I open-label, single- } \\
\text { arm, Simon two-stage } \\
\text { study }\end{array}$ & $\begin{array}{l}\text { Trastuzumab plus pertuzumab at } \\
\text { standard doses }\end{array}$ & - Two partial responses & 34 \\
\hline $\begin{array}{l}\text { BOI } 7929 \\
n=66 \\
n=29\end{array}$ & $\begin{array}{l}\text { HER2-positive } \\
\text { metastatic breast cancer } \\
\text { after standard therapy }\end{array}$ & $\begin{array}{l}\text { Phase II multicenter, } \\
\text { open-label, single-arm, } \\
\text { Simon two-stage study } \\
\text { Third cohort }\end{array}$ & $\begin{array}{l}\text { Trastuzumab plus pertuzumab } \\
\text { Pertuzumab alone }\end{array}$ & $\begin{array}{l}\text { - Objective response } \\
\text { rate: } 24.2 \% \\
\text { - Clinical benefit rate: } 50 \% \\
\text { - Complete response: } \\
7.6 \% \\
\text { - Partial response: } 16.7 \% \\
\text { - Pertuzumab active } \\
\text { but combination of } \\
\text { trastuzumab and } \\
\text { pertuzumab better }\end{array}$ & 18 \\
\hline $\begin{array}{l}\text { CLEOPATRA } \\
\mathrm{n}=808\end{array}$ & $\begin{array}{l}\text { First-line HER2- } \\
\text { positive metastatic } \\
\text { breast cancer }\end{array}$ & $\begin{array}{l}\text { Phase III randomized, } \\
\text { double-blind, placebo- } \\
\text { controlled, }\end{array}$ & $\begin{array}{l}\text { I:I randomization } \\
\text { A) Placebo plus trastuzumab plus } \\
\text { docetaxel } \\
\text { B) Pertuzmab plus trastuzumab } \\
\text { plus docetaxel }\end{array}$ & $\begin{array}{l}\text { - Median PFS was I2.4 } \\
\text { months vs } 18.5 \text { months } \\
\text { in the pertuzumab } \\
\text { group (HR for } \\
\text { progression or death, } \\
0.62 ; P<0.00 \mathrm{I} \text { ) }\end{array}$ & 21 \\
\hline $\begin{array}{l}\text { TRYPHAENA } \\
\mathrm{n}=225\end{array}$ & $\begin{array}{l}\text { Locally advanced, } \\
\text { inflammatory or early } \\
\text { stage HER2-positive } \\
\text { breast cancer }\end{array}$ & $\begin{array}{l}\text { Phase II randomized } \\
\text { neoadjuvant, } \\
\text { noncomparative }\end{array}$ & $\begin{array}{l}\text { I:I:I randomization } \\
\text { A) Concurrent }(n=75) \\
\text { FEC }+ \text { trastuzumab }+ \\
\text { pertuzumab } \times 3 \text {, } \\
\rightarrow \text { docetaxel }+ \\
\text { trastuzumab }+ \text { pertuzumb } \times 3 \\
\text { B) Sequential }(n=75) \\
\text { FEC } \times 3 \rightarrow \text { trastuzumab }+ \\
\text { pertuzumab }+ \text { docetaxel } \times 3 \\
\text { C) Anthracycline-free }(n=75) \\
\text { Carboplatin }+ \text { docetaxel }+ \\
\text { trastuzumab }+ \text { pertuzumab } \times 6\end{array}$ & $\begin{array}{l}\text { Regardless of } \\
\text { chemotherapy chosen, } \\
\text { the combination } \\
\text { of pertuzumab and } \\
\text { trastuzumab resulted } \\
\text { in high PCR rates } \\
(57 \%-66 \%)\end{array}$ & 20 \\
\hline $\begin{array}{l}\text { NEOSPHERE } \\
\mathrm{n}=417\end{array}$ & $\begin{array}{l}\text { Locally advanced, } \\
\text { inflammatory or early } \\
\text { stage HER2-positive } \\
\text { breast cancer }\end{array}$ & $\begin{array}{l}\text { Phase II randomized } \\
\text { neoadjuvant multicenter, } \\
\text { open-label }\end{array}$ & $\begin{array}{l}\text { I:I:I:I randomization } \\
\text { A) trastuzumab + docetaxel } \\
\text { B) pertuzumab + trastuzumab + } \\
\text { docetaxel } \\
\text { C) pertuzumab + trastuzumab } \\
\text { D) pertuzumab + docetaxel }\end{array}$ & $\begin{array}{l}\text { - Group B significantly } \\
\text { improved pathological } \\
\text { complete response rate } \\
45.8 \% \\
\text { - Antitumor activity in } \\
\text { group C - minimal } \\
\text { toxicity }\end{array}$ & 19 \\
\hline
\end{tabular}

Abbreviations: FEC, 5-fluorouracil, epirubicin, cyclophosphamide; HR, hazard ratio; PFS, progression-free survival.

but was significantly lower in group $\mathrm{C}$ (four serious AEs in $4 \%$ of patients). The study concluded that patients given pertuzumab and trastuzumab plus docetaxel (group B) had a significantly improved pathological complete response rate compared with those given trastuzumab plus docetaxel alone, which indicates that a chemotherapy backbone is necessary to improve the $\mathrm{pCR}$ rate.

The efficacy of neoadjuvant pertuzumab was confirmed by the recently presented TRYPHAENA Study. ${ }^{20}$ The trial examined patients with newly diagnosed HER2-positive early breast cancer and investigated three experimental neoadjuvant regimens. The common feature of all three regimens was the combination of pertuzumab and trastu- zumab for at least three cycles. Each regimen combined these two HER2-directed antibodies with anthracyclinetaxane-based or carboplatin-taxane-based standard chemotherapy backbones either concomitantly or sequentially for six cycles. Following surgery, all patients received 1 year of adjuvant trastuzumab. The primary endpoint was assessment of the safety and tolerability of neoadjuvant treatment. During neoadjuvant treatment, the left ventricular ejection fraction was monitored on a regular basis. The key secondary endpoint was the pCR rate Approximately 75 patients were randomized to each of the three neoadjuvant regimens, and all were well tolerated with no symptomatic cardiac toxicity even if both HER2-directed antibodies were 
given concomitantly with anthracyclines. Treatment with each regimen yielded impressive $\mathrm{pCR}$ rates, ranging from $45 \%$ to $66 \%$ with little difference between them. However, pCR rates were substantially higher in patients with ERnegative and progesterone receptor (PR)-negative tumors (up to $83.8 \%$ ), compared to patients with ER-negative and/or PR-positive tumors. These findings, together with the previously reported results of the NEOSPHERE trial described above, clearly illustrate the potential benefits of dual HER2 inhibition using pertuzumab and trastuzumab; however, the long-term benefits of the neoadjuvant regimens investigated in the TRYPHAENA trial are still unknown. At the time of the writing, only $16 \%$ of patients had completed adjuvant therapy.

Based on the encouraging phase II data, a randomized Phase III trial has begun that enrolled women with metastatic HER2/neu positive breast cancer in first-line treatment consisting of docetaxel and trastuzumab with placebo or pertuzumab. ${ }^{21}$ In CLEOPATRA (Clinical Evaluation of Pertuzumab and Trastuzumab), an international, Phase III, doubleblind, randomized registration trial, 808 patients (mean age, 54 years) received either placebo plus trastuzumab (an 8-mg/ $\mathrm{kg}$ loading dose, followed by a 6-mg/kg maintenance dose) plus docetaxel $\left(75 \mathrm{mg} / \mathrm{m}^{2}\right.$, escalating to $100 \mathrm{mg} / \mathrm{m}^{2}$ if tolerated, for six cycles or more, as recommended) or pertuzumab (an 840-mg loading dose, followed by a 420-mg maintenance dose) plus trastuzumab (an $8-\mathrm{mg} / \mathrm{kg}$ loading dose, followed by a $6-\mathrm{mg} / \mathrm{kg}$ maintenance dose). ${ }^{21}$ Patients were allowed to receive one previous hormonal treatment for metastatic breast cancer and/or prior systemic neoadjuvant or adjuvant therapy, including prior trastuzumab and taxanes. PFS (the primary endpoint) was reported at a median of 18.5 months with pertuzumab/trastuzumab/docetaxel and at 12.4 months with placebo/trastuzumab/docetaxel (6.1 months; hazard ratio $[\mathrm{HR}]=0.62 ; 95 \% \mathrm{CI}: 0.51-0.75 ; P<0.0001)$. Calling the magnitude of effects with the pertuzumab combination "unprecedented," the authors speculated that the new regimen could be a practice-changing discovery for the treatment of patients with HER2-positive, first-line metastatic breast cancer. Subgroup analyses revealed that the beneficial effect of pertuzumab on PFS occurred regardless of the patients' prior exposure to adjuvant or neoadjuvant chemotherapy, their geographic region, their hormone receptor status (ie, estrogen and/or progesterone receptor-positive or estrogen and progesterone-receptor negative), or their HER2 positivity, which was assessed by immunohistochemistry (membrane staining $3+$ ) or by fluorescence in situ hybridization (amplificaton of the HER2 gene).
In terms of patient age, progression-free survival hazard ratios significantly favored pertuzumab in patients aged $<65$ or $\geq 65$ years, and patients aged $<75$ years, but not in those aged $\geq 75$ years, where the HR favored pertuzumab but the number of patients was very small $(n=19)$. The interim analysis of overall survival was performed after 165 events ( $43 \%$ of the prespecified total number for the final analysis) had occurred. More deaths occurred in the control group than in the pertuzumab group (96 [23.6\%] vs 69 [17.2\%]). The hazard ratio was 0.64 ( $95 \% \mathrm{CI}: 0.47$ to $0.88 ; P=0.005$ ), which did not meet the O'Brien-Fleming stopping boundary for this interim analysis of overall survival (HR $\leq 0.603$; $P \leq 0.0012)$ and was, therefore, not significant. However, the data showed a strong trend toward a survival benefit with the pertuzumab-trastuzumab-docetaxel therapy. ${ }^{21}$

\section{Ongoing trials with pertuzumab}

Several other trials are examining the efficacy of pertuzumab in patients with early breast and advanced breast cancer (see Table 3). Both, in vitro and in vivo studies suggest that the combination of pertuzumab and trastuzumab has synergistic activity in breast cancer; therefore, clinical trials are now focusing on the combination of both antibodies. ${ }^{22}$ For example, the PHEREXA study (NCT01026142) is designed to investigate the potential benefit of administering pertuzumab and trastuzmab combined with capecitabine in patients with HER2-positive metastatic breast cancer whose disease has progressed during or following trastuzumab-based therapy for first-line metastatic breast cancer. ${ }^{23}$ In this multicenter, openlabel, Phase II trial, patients are randomized 1:1 to receive trastuzumab in combination with capecitabine or trastuzumab, pertuzumab, and capecitabine. Enrollment began in January 2010 and 450 patients will be recruited from $~ 130$ sites in 19 countries. The primary endpoint is PFS (independent assessment). Secondary endpoints include overall survival, PFS (investigator assessment), and safety/tolerability. A panel of biomarkers including HER1, HER2, and HER3 receptor status and downstream markers will be analyzed.

The Phase III MARIANNE trial (NCT01120184) is currently underway and is comparing the efficacy of the investigational immunoconjugate trastuzumab-emtansine (trastuzumab-DM1) with or without pertuzumab with that of trastuzumab plus a taxane for the first-line treatment of patients with HER2-positive locally advanced or metastatic breast cancer. ${ }^{24} \mathrm{~T}$-DM1 is a unique antibody-drug conjugate (ADC) composed of trastuzumab, a stable thioether linker, and the highly potent cytotoxic agent, DM1 (derivative of maytansine). In a randomized Phase II trial, single-agent 


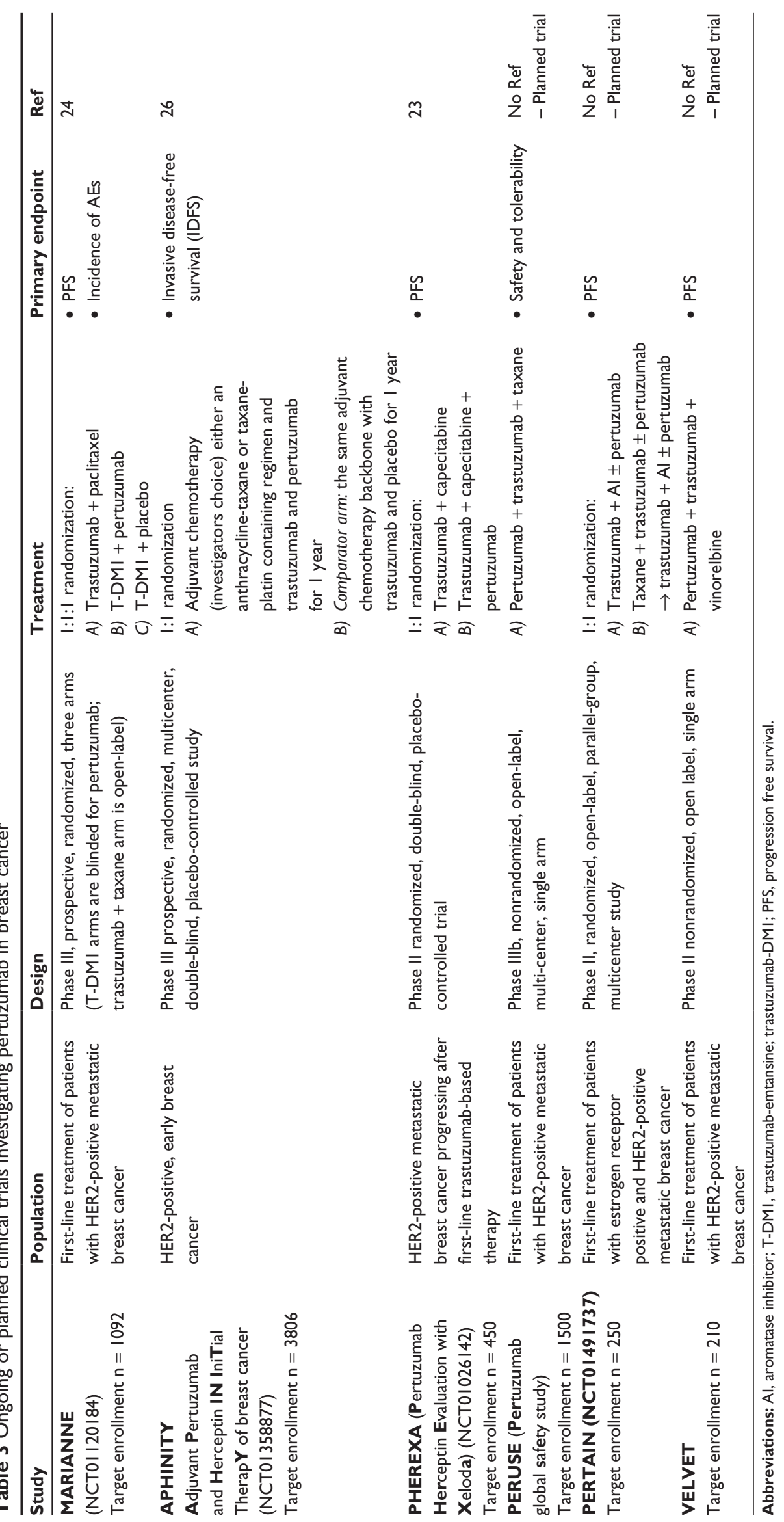


T-DM1 had comparable efficacy to trastuzumab plus docetaxel in the first-line treatment of HER2-positive locally advanced or metastatic breast cancer. ${ }^{25}$ T-DM1 and pertuzumab bind to different epitopes on HER2, and have distinct mechanisms of action, which suggests that the combination may result in a more complete blockade of HER2. T-DM1 plus pertuzumab showed synergistic antitumor effects in preclinical models, and the preliminary Phase $\mathrm{Ib} / \mathrm{II}$ trial results showed acceptable tolerability and promising efficacy in patients with metastatic breast cancer. ${ }^{13}$ This systemic chemotherapy-sparing combination has the potential to establish a new treatment paradigm by optimizing efficacy while minimizing toxicity. Patients are randomized 1:1:1 to each arm. The primary endpoint is PFS. Secondary endpoints include safety, overall response rate, overall survival, duration of response and quality of life. Target enrollment is 1092 patients across $\sim 310$ sites globally.

Finally, the Adjuvant Pertuzumab and Herceptin IN IniTial TherapY of Breast Cancer (APHINITY) trial (NCT01358877) is examining the efficacy of pertuzumab plus trastuzumab, compared to that of trastuzumab alone in patients receiving adjuvant chemotherapy for HER2-positive, nonmetastatic breast cancer. ${ }^{26}$ While the adjuvant use of trastuzumab has been shown to improve disease-free and overall survival, not all patients treated with this agent benefit from this therapy. APHINITY is a prospective, randomized, multicenter, doubleblind, placebo-controlled study in patients with HER2-positive early breast cancer. Patients will be randomized to one of two arms (1:1 ratio). The investigational arm will be comprised of a course of adjuvant CT (investigators choice) consisting of either an anthracycline-taxane or a taxane-platin that contains regimens, trastuzumab, and pertuzumab for 1 year. The comparator arm will consist of the same adjuvant CT backbone with trastuzumab and placebo for 1 year.

\section{Adverse events and cardiac safety of pertuzumab}

HER 2 and HER 4 are crucial for mouse embryonic heart development, and studies have shown that HER2 expression is required for the development of ventricular muscles and valves in rodents, while the activation of HER2 promotes cardiomyocyte survival. ${ }^{27-30}$ Trastuzumab may cause an asymptomatic decline in the left ventricular ejection fraction (LVEF), known as left ventricular systolic dysfunction (LVSD) ${ }^{21}$ However, symptomatic heart failure (HF) under trastuzumab is relatively uncommon. Nevertheless, due to the known effects of trastuzumab on the human heart, it is of major concern that pertuzumab may cause similar or worse declines in cardiac functions. The results of a recently published meta-analysis of 14 Phase II trials, which included 598 patients, demonstrated that pertuzumab (exposure ranging from a median of three cycles to a median of 26 cycles) was generally well tolerated. ${ }^{31}$ The incidence of LVSD was low, with most events being asymptomatic and detected at scheduled evaluations. The median timing of LVSD and HF was around cycle 4 (range 1-15) with 34/39 (87\%) events occurring between cycles 1 and 7 . Additionally, when pertuzumab was administered in combination with trastuzumab or nonanthracycline-containing cytotoxic chemotherapy, there was no marked increase in observed cardiac dysfunction. In this analysis of 598 unique patients exposed to pertuzumab, 35 (5.9\%) cases of asymptomatic LVSD and four $(0.7 \%)$ cases of symptomatic HF were reported. ${ }^{31}$ Results from the completed NEOSPHERE, TRYPHAENA, and CLEOPATRA trials also indicate a low incidence of symptomatic and asymptomatic LVSD. ${ }^{19-21}$

Gastrointestinal toxicities (diarrhea, nausea, vomiting, and abdominal pain) and fatigue are the most frequently reported AEs associated with single-agent therapy. Diarrhea and rash are common events that increased with pertuzumab in combination with chemotherapy, compared with chemotherapy alone. The most common AEs during dual therapy with pertuzumab and trastuzumab in metastatic breast cancer (BO17929) were diarrhea, fatigue, nausea, and rash. ${ }^{18}$ The majority of these AEs were NCI-CTC grade 1 or 2 in severity. The mechanisms behind diarrhea and rash are unknown, but similar side effects are observed with other agents that cause HER1 inhibition. The most frequently occurring AEs during neoadjuvant treatment of patients with locally advanced breast cancer, using pertuzumab and trastuzumab in combination with chemotherapy, were alopecia, neutropenia, diarrhea, nausea, fatigue, rash, and mucosal inflammation. ${ }^{19,20}$

Adding pertuzumab to the trastuzumab plus docetaxel regimen did not notably affect the overall safety profile, and the tolerability of pertuzumab plus docetaxel was also broadly comparable to the triple regimen. ${ }^{32}$ Patients receiving trastuzumab and pertuzumab without docetaxel in the neoadjuvant setting reported notably fewer AEs across most body systems, compared to patients who received treatments containing chemotherapy. Serious or severe infusion-related symptoms have been rarely observed in patients $(<1 \%)$ receiving pertuzumab.

\section{Conclusion}

The HER family of receptor tyrosine kinases is an important mediator in cancer development and progression, and 
treatment directed against individual members of this family is a successful strategy for cancer therapy. As a HER dimerization inhibitor, pertuzumab promises to be widely applicable across tumor types, particularly since it is hypothesized that HER2/neu overexpression is not required for it to be an active agent. Pertuzumab has revealed itself to be a promising agent in metastatic HER2 positive breast cancer, in which a combination with trastuzumab has shown impressive activity among women who had otherwise progressed on trastuzumabbased therapy. The antibody has also shown excellent efficacy in combination with trastuzumab and chemotherapy in a neoadjuvant setting. However, no head-to-head comparison of trastuzumab and pertuzumab exists, which is a weakness of the current data. Therefore, it is unknown if single agent activity of pertuzumab is comparable to trastuzumab. On the other hand, single-agent activity of pertuzumab without any chemotherapy backbone has proved to be very limited even in HER2 positive breast cancer. This observation has halted the further investigation of single-agent pertuzumab in breast cancer. Since HER2 overexpression serves as a predictor of trastuzumab activity, it is necessary to search for the biologic correlate that predicts the activity of pertuzumab. Nevertheless, pertuzumab remains one of the most promising agents in the treatment of HER2-positive breast cancer. Clinical trials are currently recruiting subjects to investigate pertuzumab using new regimes that may discontinue the use of chemotherapeutic agents in the future.

\section{Disclosure}

The authors report no conflicts of interest in this work.

\section{References}

1. Cobleigh MA, Vogel CL, Tripathy D, et al. Multinational study of the efficacy and safety of humanized anti-HER2 monoclonal antibody in women who have HER2-overexpressing metastatic breast cancer that has progressed after chemotherapy for metastatic disease. J Clin Oncol. 1999;17(9):2639-2648.

2. Slamon D, Eiermann W, Robert N, et al. Adjuvant trastuzumab in HER2positive breast cancer. N Engl J Med. 2011;365(14):1273-1283.

3. Baselga J. Treatment of HER2-overexpressing breast cancer. Ann Oncol. 2010;21 Suppl 7:vii36-vii40.

4. Slamon DJ, Leyland-Jones B, Shak S, et al. Use of chemotherapy plus a monoclonal antibody against HER2 for metastatic breast cancer that overexpresses HER2. N Engl J Med. 2001;344(11):783-792.

5. Hubalek M, Brunner C, Mattha K, Marth C. Resistance to HER2-targeted therapy: mechanisms of trastuzumab resistance and possible strategies to overcome unresponsiveness to treatment. Wien Med Wochenschr. 2010;160(19-20):506-512.

6. Lee-Hoeflich ST, Crocker L, Yao E, et al. A central role for HER3 in HER2-amplified breast cancer: implications for targeted therapy. Cancer Res. 2008;68(14):5878-5887.

7. Diermeier-Daucher S, Hasmann M, Brockhoff G. Flow cytometric FRET analysis of erbB receptor interaction on a cell-by-cell basis. Ann NY Acad Sci. 2008;1130:280-286.
8. Franklin MC, Carey KD, Vajdos FF, Leahy DJ, de Vos AM, Sliwkowski MX. Insights into ErbB signaling from the structure of the ErbB2-pertuzumab complex. Cancer Cell. 2004;5(4):317-328.

9. Cho HS, Mason K, Ramyar KX, et al. Structure of the extracellular region of HER 2 alone and in complex with the Herceptin Fab. Nature. 2003;421(6924):756-760.

10. Agus DB, Akita RW, Fox WD, et al. Targeting ligand-activated ErbB2 signaling inhibits breast and prostate tumor growth. Cancer Cell. 2002;2(2):127-137.

11. Scheuer W, Friess T, Burtscher H, Bossenmaier B, Endl J, Hasmann M. Strongly enhanced antitumor activity of trastuzumab and pertuzumab combination treatment on HER2-positive human xenograft tumor models. Cancer Res. 2009;69(24):9330-9336.

12. Friess T, Scheuer W, Hasmann M. Combination treatment with erlotinib and pertuzumab against human tumor xenografts is superior to monotherapy. Clin Cancer Res. 2005;11(14):5300-5309.

13. LoRusso PM, Weiss D, Guardino E, Girish S, Sliwkowski MX. Trastuzumab emtansine: a unique antibody-drug conjugate in development for human epidermal growth factor receptor 2-positive cancer. Clin Cancer Res. 2011;17(20):6437-6447.

14. Agus DB, Gordon MS, Taylor C, et al. Phase I clinical study of pertuzumab, a novel HER dimerization inhibitor, in patients with advanced cancer. J Clin Oncol. 2005;23(11):2534-2543.

15. Adams CW, Allison DE, Flagella K, et al. Humanization of a recombinant monoclonal antibody to produce a therapeutic HER dimerization inhibitor, pertuzumab. Cancer Immunol Immunother. 2006;55(6):717-727.

16. Ng CM, Lum BL, Gimenez V, Kelsey S, Allison D. Rationale for fixed dosing of pertuzumab in cancer patients based on population pharmacokinetic analysis. Pharm Res. 2006;23(6):1275-1284.

17. Gianni L, Llado A, Bianchi G, et al. Open-label, phase II, multicenter, randomized study of the efficacy and safety of two dose levels of pertuzumab, a human epidermal growth factor receptor 2 dimerization inhibitor, in patients with human epidermal growth factor receptor 2-negative metastatic breast cancer. J Clin Oncol. 2010;28(7):1131-1137.

18. Baselga J, Gelmon KA, Verma S, et al. Phase II trial of pertuzumab and trastuzumab in patients with human epidermal growth factor receptor 2-positive metastatic breast cancer that progressed during prior trastuzumab therapy. J Clin Oncol. 2010;28(7):1138-1144.

19. Gianni L, Pienkowski T, Im YH, et al. Efficacy and safety of neoadjuvant pertuzumab and trastuzumab in women with locally advanced, inflammatory, or early HER2-positive breast cancer (NeoSphere): a randomised multicentre, open-label, phase 2 trial. Lancet Oncol. 2012;13(1):25-32.

20. Schneeweiss A. Neoadjuvant pertuzumab and trastuzumab concurrent or sequential with an anthracycline containing or concurrent with an anthracycline-free standard regimen: a randomized phase II study (TRYPHAENA). Paper presented at the 2011 San Antonio Breast Cancer Symposium; December 6-10, 2011; San Antonio, TX. Abstract S5-S6. Cancer Res. 2011.

21. Baselga J, Cortes J, Kim SB, et al. Pertuzumab plus trastuzumab plus docetaxel for metastatic breast cancer. N Engl J Med. 2012; 366(2):109-119.

22. Nahta R, Hung MC, Esteva FJ. The HER-2-targeting antibodies trastuzumab and pertuzumab synergistically inhibit the survival of breast cancer cells. Cancer Res. 2004;64(7):2343-2346.

23. Munoz-Mateu M, Urruticoechea A, Separovic R, et al. Trastuzumab plus capecitabine with or without pertuzumab in patients with HER2-positive MBC whose disease has progressed during or following trastuzumab-based therapy for first-line metastatic disease: a multicenter, randomized, two-arm, phase II study (PHEREXA). J Clin Oncol. 2011;29 Suppl:Abstr TPS118.

24. Ellis PA, Barrios CH, Im Y, Patre M, Branle F, Perez EA. MARIANNE: a phase III, randomized study of trastuzumab-DM1 (T-DM1) with or without pertuzumab $(\mathrm{P})$ compared with trastuzumab $(\mathrm{H})$ plus taxane for first-line treatment of HER2-positive, progressive, or recurrent locally advanced or metastatic breast cancer (MBC). J Clin Oncol. 2011;29 Suppl:Abstr TPS102. 
25. Hurvitz SA, et al. Phase II trial on the efficacy of single-agent T-DM1 compared to trastuzumab + docetaxel in the first-line treatment of HER2-positive locally advanced or MBC. Abstract 5.001: Proceedings of the European Multidisciplinary Cancer Congress (ECCO/ESMO); 2011 Sep 23-27; Stockholm, Sweden.

26. von Minckwitz G, Balsega J, Bradbury I, et al. Adjuvant pertuzumab and Herceptin in initial therapy of breast cancer: APHINITY. Cancer Res. 2011;71(24 Suppl):602S.

27. Lee KF, Simon H, Chen H, Bates B, Hung MC, Hauser C. Requirement for neuregulin receptor erbB2 in neural and cardiac development. Nature. 1995;378(6555):394-398.

28. Gassmann M, Casagranda F, Orioli D, et al. Aberrant neural and cardiac development in mice lacking the ErbB4 neuregulin receptor. Nature. 1995;378(6555):390-394.

29. Camenisch TD, Schroeder JA, Bradley J, Klewer SE, McDonald JA. Heart-valve mesenchyme formation is dependent on hyaluronanaugmented activation of ErbB2-ErbB3 receptors. Nat Med. 2002;8(8): $850-855$.
30. Zhao YY, Sawyer DR, Baliga RR, et al. Neuregulins promote survival and growth of cardiac myocytes. Persistence of ErbB2 and ErbB4 expression in neonatal and adult ventricular myocytes. J Biol Chem. 1998;273(17):10261-10269.

31. Perez EA. Cardiac toxicity of ErbB2-targeted therapies: what do we know? Clin Breast Cancer. 2008;8 Suppl 3:S114-S120.

32. Lenihan D, Suter T, Brammer M, Neate C, Ross G, Baselga J. Pooled analysis of cardiac safety in patients with cancer treated with pertuzumab. Ann Oncol. 2012;23(3):791-800.

33. Portera CC, Walshe JM, Rosing DR, et al. Cardiac toxicity and efficacy of trastuzumab combined with pertuzumab in patients with [corrected] human epidermal growth factor receptor 2-positive metastatic breast cancer. Clin Cancer Res. 2008;14(9):2710-2716.

34. Baselga J, Cortes J, Fumoleau P, et al. Pertuzumab and trastuzumab: re-responses to 2 biological agents in patients with HER2-positive breast cancer which had previously progressed during therapy with each agent given separately: a new biological and clinical observation. Cancer Res. 2009;69(24 Suppl):Abstract 5114.
Breast Cancer: Targets and Therapy

\section{Publish your work in this journal}

Breast Cancer: Targets and Therapy is an international, peerreviewed open access journal focusing on breast cancer research, identification of therapeutic targets and the optimal use of preventative and integrated treatment interventions to achieve improved outcomes, enhanced survival and quality of life for the cancer patient.

\section{Dovepress}

View the full aims and scopes of this journal here. The manuscript management system is completely online and includes a very quick and fair peer-review system, which is all easy to use. Visit http:// www.dovepress.com/testimonials.php to read real quotes from published authors

Submit your manuscript here: http://www.dovepress.com/breast-cancer---targets-and-therapy-journal 